\title{
Comparison of Spatial QRS-T Angle in Different Healthy Racial Groups
}

\author{
Elaine Clark, Peter W Macfarlane \\ University of Glasgow, Scotland
}

\begin{abstract}
The spatial QRS-T angle has been found to be an important prognostic predictor for cardiovascular mortality. The aim of this study was to compare the normal limits of the spatial QRS-T angle in four cohorts of apparently healthy, different racial groups. The University of Glasgow ECG Analysis Program was used to analyze the ECGs. The orthogonal $X, Y$ and $Z$ lead data was derived from the standard lead data, using the inverse Dower method, and the spatial QRS-T angle was calculated. Statistical analysis was undertaken using the SPSS Statistics package. Angles were calculated for 2553 males and 1649 females aged between 18 and 87 years. The mean spatial QRS-T angle for males in Black, Caucasian, Chinese and Indian populations was $67 \pm 27^{\circ}$, $69 \pm 29^{\circ}, 59 \pm 26^{\circ}$ and $71 \pm 33^{\circ}$ respectively with the values for the Chinese cohort being significantly different than the other cohorts $(P<0.001)$. For females, the corresponding mean values were $42 \pm 23^{\circ}, 57 \pm 25^{\circ}$, $40 \pm 22^{\circ}$ and $41 \pm 25^{\circ}$, with the values for the Caucasian cohort being significantly different from the others $(P<0.001)$. In all ethnic groups, the mean spatial QRS-T angle was higher in males than in females (overall mean difference of $21^{\circ}$ ). There was a wide range of upper limits of normal for the racial groups. Race and gender should be considered when assessing the spatial QRS-T angle.
\end{abstract}

\section{Introduction}

The spatial QRS-T angle is the angle between the maximum spatial QRS vector and the maximum spatial T vector (Figure 1). These measurements in threedimensional space are obtained from a vectorcardiogram. The $\mathrm{X}, \mathrm{Y}, \mathrm{Z}$ leads, which are required to obtain a vectorcardiogram, are rarely recorded routinely but can be derived from the standard 12 lead ECG, and so the spatial QRS-T angle can be obtained from the 12 lead ECG relatively easily using an automated ECG analysis program.

In the last few years, there has been renewed interest in spatial QRS-T angle as it has been found to be of prognostic value. A wide spatial QRS-T angle has been associated with higher risk of cardiac death in the elderly $[1,2]$. Indeed, Yamasaki et al found that, for a mainly male clinical population, it was the most significant predictor of cardiovascular mortality [3]. In their 2013 review, Voulgari et al published a table summarising the studies on the prognostic value of this measurement on cardiovascular morbidity and mortality [4]. They concluded that the spatial QRS-T angle is an important prognostic predictor and that it is superior to more conventional ECG parameters.

Figure 1. Illustration of a QRS vector loop and T vector loop and the resulting maximum spatial QRS vector and maximum spatial $\mathrm{T}$ vector, with the spatial QRS-T angle between them.
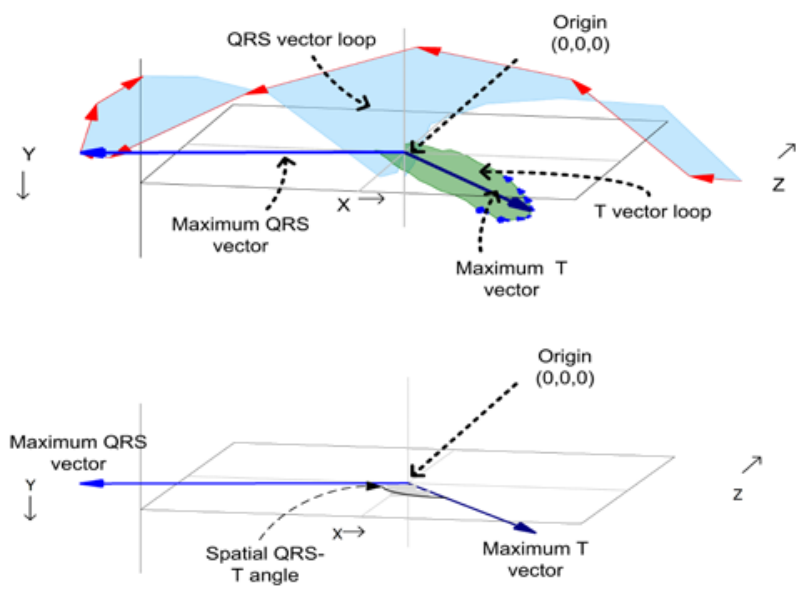

While it is known that the spatial QRS-T angle varies with age and gender [5], to our knowledge there is less information available regarding dependence on race. The present study compares the normal limits of the spatial QRS-T angle in four cohorts of apparently healthy, different racial groups. The aim was to determine if there were any significant differences due to race as well as age and gender.

\section{Methods}

Four databases of ECGs were used in the study. The ECGs have been recorded over a period of years in different parts of the world. They are all from apparently 
healthy populations, namely Black (Nigerian), Caucasian (Scottish), Chinese (Taiwanese) and Indian cohorts. These databases have been studied in relation to normal limits and have been reported separately and in comparative studies [6-9].

The ECGs were analysed using the 2016 version of the University of Glasgow ECG Analysis Program [10]. The 10 -second raw data for the 12 lead ECGs was input to the program and the orthogonal $\mathrm{X}, \mathrm{Y}$ and $\mathrm{Z}$ lead data was derived from the standard lead data, using the inverse Dower method [11].

Using the average beat data from the $\mathrm{X}, \mathrm{Y}$ and $\mathrm{Z}$ leads, the maximum spatial QRS vector, maximum spatial $\mathrm{T}$ vector and the resultant spatial QRS-T angle were calculated by the program (Figure 1).

For each cohort, the mean and standard deviation of the spatial QRS-T angle were calculated, as well as the normal range (between $2^{\text {nd }}$ and $98^{\text {th }}$ percentile). The SPSS Statistics package was used. Comparisons of differences between the groups were made using the Mann-Whitney $\mathrm{U}$ test. Analysis was also carried out with respect to subcategories of age and gender, within each racial group.

\section{Results}

A total of 4223 ECGs were analysed. The composition of the groups with respect to age and gender is given in Table 1, together with the number of ECGs excluded due to technical reasons. After exclusions, results were available for 2553 males and 1649 females.

Table 1. Numbers of males and females, average age \pm SD, and numbers excluded in each cohort.

\begin{tabular}{|c|c|c|c|c|}
\hline & $\begin{array}{l}\text { Male } \\
\text { (Age in } \\
\text { years) }\end{array}$ & $\begin{array}{l}\text { Female } \\
\text { Age in } \\
\text { years }\end{array}$ & Excluded & Total \\
\hline Black & $782(43 \pm 13)$ & $479(43 \pm 15)$ & 7 & 1254 \\
\hline Caucasian & $859(39 \pm 12)$ & $637(35 \pm 13)$ & 3 & 1493 \\
\hline Chinese & $248(43 \pm 15)$ & $255(43 \pm 14)$ & 6 & 497 \\
\hline Indian & $670(36 \pm 14)$ & $293(36 \pm 16)$ & 5 & 958 \\
\hline Total & 2559 & 1664 & 21 & 4202 \\
\hline
\end{tabular}

The statistical results for the Black, Caucasian, Chinese and Indian populations are given in Table 2.

A comparison of the differences between the ethnic groups for males showed statistical differences $(\mathrm{P}<0.05)$ with the exception of Caucasian v Black and Caucasian v Indian. In particular, the spatial QRS-T angle for Chinese males was significantly lower than for all other groups $(\mathrm{P}<0.001)$. The spatial QRS-T angle for Caucasian females was significantly higher $(\mathrm{P}<0.001)$ than for each of the other three groups. (Figure 2).

Figure 2. The upper limits of normal and mean spatial QRS-T angle values for males and females of the four racial groups (Black, Caucasian, Chinese and Indian).

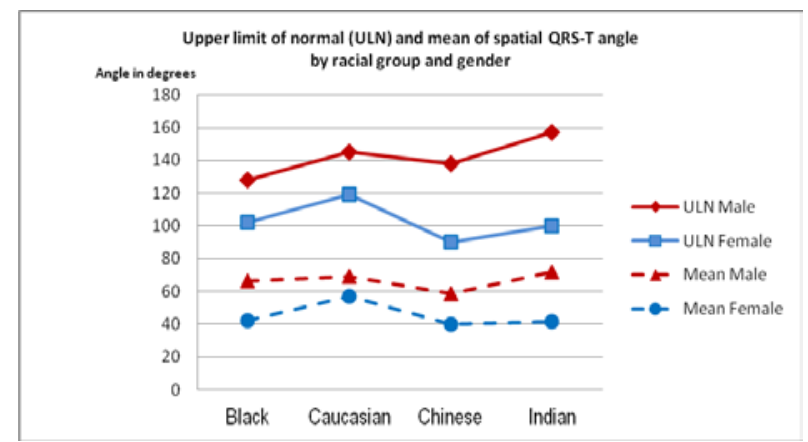

In all ethnic groups, the age-independent mean spatial QRS-T angle was higher in males than in females, with an overall mean difference of $21^{\circ}$. In each age-group, the mean spatial QRS-T angle was higher in males than in females for each of the cohorts. The values with respect to age-groups are shown in the graphs in Figure 3.

Figure 3. The mean spatial QRS-T angle for males and females by age-group for each of the four racial groups (Black, Caucasian, Chinese and Indian).

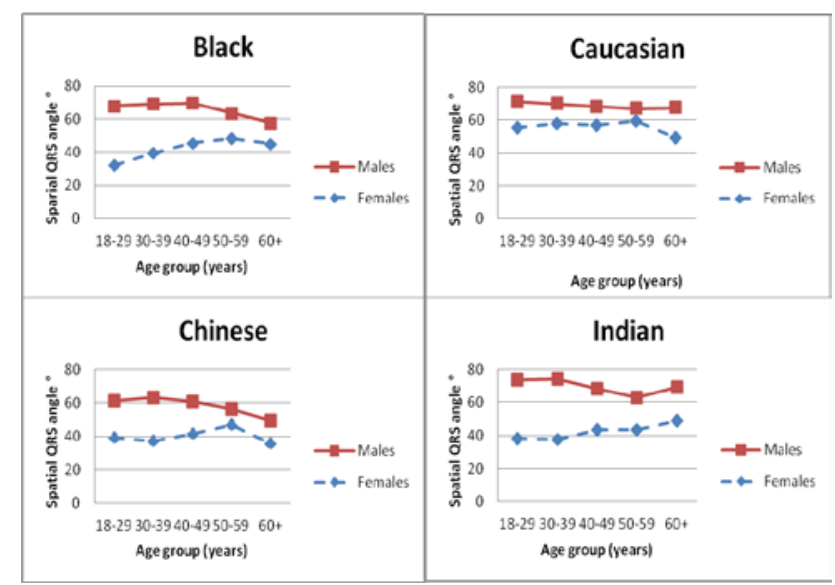

Table 2 . Mean, standard deviation, $2^{\text {nd }}$ to $98^{\text {th }}$ percentile ranges and totals for four cohorts.

\begin{tabular}{lllllllll}
\hline QRS-T angle & \multicolumn{2}{c}{ Black } & \multicolumn{2}{c}{ Caucasian } & \multicolumn{2}{c}{ Chinese } & \multicolumn{2}{c}{ Indian } \\
& Male & Female & Male & Female & Male & Female & Male & Female \\
\hline Mean(SD) & (67(27) & $42(23)$ & $69(29)$ & $57(25)$ & $59(26)$ & $40(22)$ & $71(33)$ & $41(25)$ \\
$2 \%-98 \%$ & $20-128$ & $8-102$ & $16-145$ & $13-119$ & $13-138$ & $8-90$ & $16-157$ & $8-100$ \\
Total & $\mathrm{n}=779$ & $\mathrm{n}=475$ & $\mathrm{n}=858$ & $\mathrm{n}=635$ & $\mathrm{n}=248$ & $\mathrm{n}=249$ & $\mathrm{n}=668$ & $\mathrm{n}=290$ \\
\hline
\end{tabular}


It can be seen that, in general, the mean spatial QRS-T angle decreased as age increased for males. For females there was a slight increase over age-groups 30-39 years, 40-49 years, and 50-59 years. This tailed off in the $60+$ years age-group but there were fewer subjects in this age-group. For each of the age-groups, the mean spatial QRS-T angle for Chinese males was lower than for the other cohorts of corresponding age-group, and for females, the value for the Caucasian group was higher than for the other cohorts in the corresponding age-group. The spatial QRS-T angle in the Caucasian cohort was significantly different $(\mathrm{P}<0.01)$ from each of the other cohorts in all age-groups except for the 60+age-group.

\section{Discussion}

It is known that the mean spatial QRS-T angle is, in general, higher for males than females [12]. There is a wide spectrum of normal limits for spatial QRS-T angle in the literature. They vary according to the method used to derive the angle as well as the populations used in the studies. An example of a case with a wide QRS-T angle for a male of 77 years is shown in Figure 4. The statement "lateral $\mathrm{T}$ wave abnormality is nonspecific" was reported on this ECG. This is an example of a borderline case where the additional information of a spatial QRS-T angle of $164^{\circ}$, which lies outwith the overall normal limits, would have been useful. The race was unknown for this subject who did not belong to the study cohort. The angle value is above the normal limits for all the racial cohorts. Two views, from different perspectives, of the QRS and $\mathrm{T}$ vector loops, maximum vectors and spatial QRS-T angle, are shown in Figure 5.

It was found that the mean spatial QRS angle is higher for males than females in each of the ethnic groups. The upper limits of normal obtained for Caucasians in this study for the age-group 18-29 years were $108^{\circ}$ for females and $127^{\circ}$ for males. These are in line with those reported by Sherptong et al [13] in their study on a population of students in the same age-group in Leiden, Netherlands, i.e. $116^{\circ}$ for females and $130^{\circ}$ for males. They also used the inverse Dower method of matrix transformation to derive the $\mathrm{X}, \mathrm{Y}$ and $\mathrm{Z}$ lead data and hence the spatial QRS-T angle.

Both the mean spatial QRS-T angle and the upper limit of normal for Caucasian women were higher than in the other female cohorts. The difference in the upper limits of normal for the cohorts may be worth further investigation as a wide spatial QRS-T angle in post-menopausal women was found to have nearly a 3-fold increase of risk of all-cause mortality in subjects with baseline cardiovascular disease [14].

In an early study [15], it was found that the spatial QRS-T angle is associated with height, weight and position of the heart and these may be factors that influence the normal limits found for the different cohorts in the present study. We did not undertake such an analysis.

Figure 4. Average beats for the 12 standard leads and the $\mathrm{X}, \mathrm{Y}$ and $(-\mathrm{Z})$ leads from the ECG of a 77 year old male.

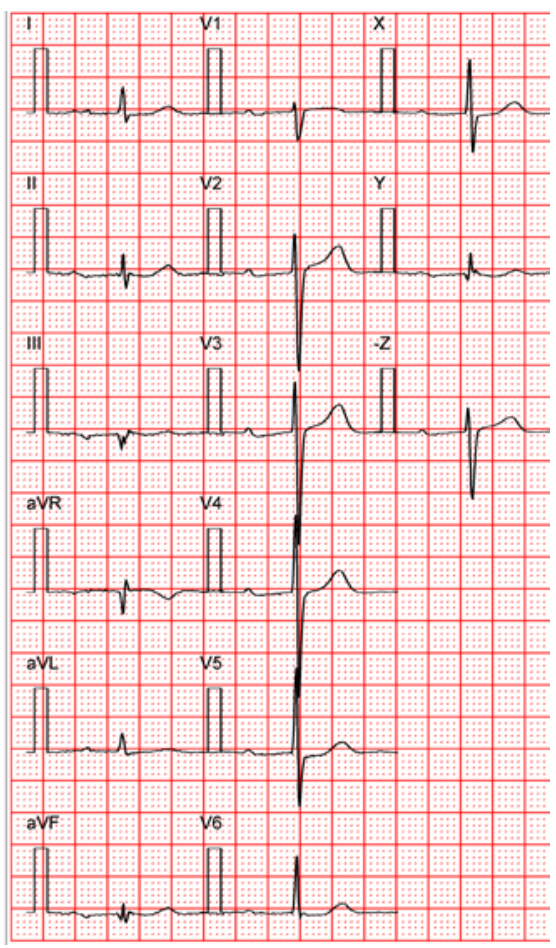

Figure 5. Views of the QRS and T vector loops for the same ECG, as in Figure 4, of a 77-year old male.
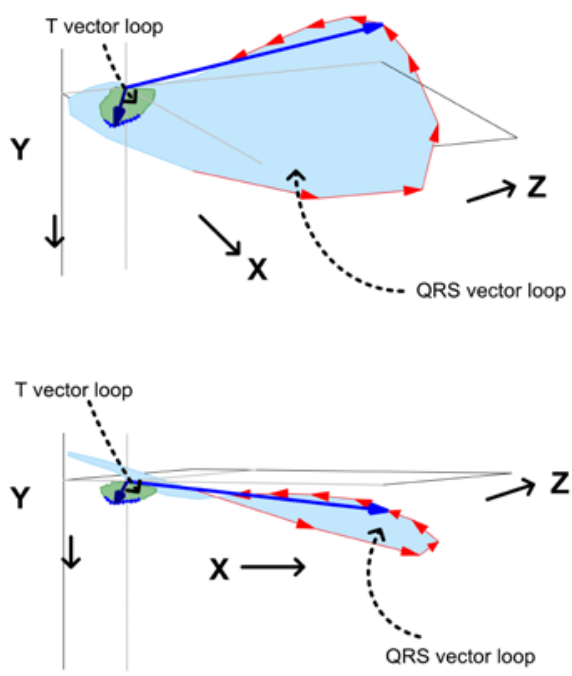
In this study, for male subjects, the upper limit of normal for the black cohort was the lowest of the four groups. This is in contrast to the findings of Whang et al [2]. In their population-based study, they found that if an abnormally wide spatial QRS-T angle was present, the subject was more likely to be older and a non-Hispanic black. This may be due to the different method of obtaining the spatial QRS-T angle. Whang et al used an algorithm which uses 3 QRS and $3 \mathrm{~T}$ wave peak-to-peak amplitudes. It may also be due to the different ethnic grouping, with the Black cohort in the present study being of Nigerian nationality compared to a black North American cohort in the study of Whang et al [2].

\section{Conclusion}

The spatial QRS-T angle is acknowledged to be a strong predictor of cardiac mortality. It can be calculated from the 12 lead ECG and hence output on the report of an automated analysis of a standard 12 lead ECG. It adds 3-dimensional information to the standard 12 lead ECG report, giving further knowledge of the direction of the cardiac depolarization and repolarization forces. While, it is a difficult task to visualise spatial vectors from the standard 12 lead ECG, automated analysis of this measurement allows its use in routine practice.

The main finding of this study with respect to race was that, for males, the spatial QRS-T angle was significantly different for the Chinese group than the other groups and that, for females, the spatial QRS-T angle was significantly different for the Caucasian group. There were large differences between the upper limits of normal for the racial groups (Table 2), e.g. a limit of $128^{\circ}$ for the Black male cohort and $157^{\circ}$ for the Indian male cohort. These different limits of normal for the racial cohorts (Figure 2) suggest that there is sufficient justification to use race and gender in the criteria for an abnormal spatial QRS-T angle.

\section{References}

[1] Kardys I, Kors J, van der Meer I, Hofman A, van der Kuip DAM, Witteman JCM. Spatial QRS-T angle predicts cardiac death in a general population. Eur Heart $\mathrm{J}$ 2003;24:1357-64.

[2] Whang W, Shimbo D, Levitan EB,Newman JD, Rautaharju PM, Davidson KW et al. Relations between QRS|T Angle, Cardiac Risk Factors, and Mortality in the Third National Health and Nutrition Examination Survey (NHANES III). Am J Cardiol 2012;109:981-7.

[3] Yamazaki T, Froelicher VF, Myers J, Chun S, Wang P. Spatial QRS-T angle predicts cardiac death in a clinical population. Heart Rhythm 2005;2:73-8.

[4] Voulgari C, Pagoni S, Tesfaye S, Tentolouris N. The Spatial QRS-T Angle: Implications in Clinical Practice. Current Cardiology Reviews 2013;9:197-210.

[5] Oehler A, Feldman T, Henrikson CA, Tereshchenko LG.
QRS-T angle: a review. Ann Noninvasive Electrocardiol 2014;19:534-42.

[6] Macfarlane PW, Lawrie TDV: The Normal Electrocardiogram and Vectorcardiogram. In Macfarlane PW, van Oosterom A, Pahlm O, Kligfield P, Janse J, Camm J (Eds): Comprehensive Electrocardiology. 2nd Ed. London: Springer-Verlag, 2011:Vol 2 483-546.

[7] Macfarlane PW, Katibi IA, Hamde ST, Singh D, Clark E, Devine B, et al. Racial Differences in the ECG - selected aspects. J Electrocardiol 2014;47:809-14.

[8] Katibi IA, Clark EN, Devine B, Lloyd SM, Macfarlane PW. Normal limits of the electrocardiogram in Nigerians. J Electrocardiol 2013;46:289-295.

[9] Chen, CY, Chiang, BN, Macfarlane, PW. Normal limits of the electrocardiogram in a Chinese population. J Electrocardiol 1989;22:1-15.

[10] Macfarlane PW, Devine B, Clark E. The University of Glasgow (Uni-G) ECG analysis program. Computers in Cardiology 2005;32:451-4.

[11] Edenbrandt L, Pahlm O. Vectorcardiogram synthesized from a 12 lead ECG: superiority of the inverse Dower matrix. J Electrocardiol 1988;21:361-7.

[12] Zhang ZM, Prineas RJ, Case D, Soliman EZ, Rautaharju $\mathrm{PM}$. Comparison of the prognostic significance of the electrocardiographic QRS/T angles in predicting incident coronary heart disease and total mortality (from the atherosclerosis risk in communities study). Am J Cardiol 2007;100:844-9.

[13] Scherptong RW, Henkens IR, Man SC, Le Cessie S, Vliegen W, Draisma HHM et al. Normal limits of the spatial QRS-T angle and ventricular gradient in 12-lead electrocardiograms of young adults: dependence on sex and heart rate. J Electrocardiol 2008;41:648-55.

[14] Rautaharju PM, Kooperberg C, Larson JC, LaCroix A. Electrocardiographic predictors of incident congestive heart failure and all-cause mortality in postmenopausal women: the Women's Health Initiative. Circulation 2006;113:481-9.

[15] Simonson E, Keys A. The Spatial QRS and T Vector in 178 normal middle-aged men; body weight, height, relationship of QRS and T and preliminary standards. Circulation 1954; 9: 105-14.

Address for correspondence.

Elaine Clark

Institute of Health and Wellbeing

Electrocardiology Section, Level 3, New Lister Building,

Royal Infirmary

Glasgow

G31 2ER

Elaine.clark@glasgow.ac.uk 